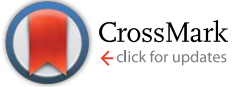

Cite this: Chem. Sci., 2015, 6, 6949

\title{
Enabling high-mobility, ambipolar charge- transport in a DPP-benzotriazole copolymer by side-chain engineering $\uparrow$
}

\author{
Mathias Gruber, $\dot{\dagger}^{\mathrm{a}}$ Seok-Heon Jung, $\dot{t}^{\mathrm{b}}$ Sam Schott, ${ }^{a}$ Deepak Venkateshvaran, ${ }^{a}$ \\ Auke Jisk Kronemeijer, ${ }^{a}$ Jens Wenzel Andreasen, ${ }^{c}$ Christopher R. McNeill, ${ }^{d}$ \\ Wallace W. H. Wong, ${ }^{e}$ Munazza Shahid, ${ }^{f}$ Martin Heeney, ${ }^{f}$ Jin-Kyun Lee ${ }^{\star b}$ \\ and Henning Sirringhaus*a
}

In this article we discuss the synthesis of four new low band-gap co-polymers based on the diketopyrrolopyrrole (DPP) and benzotriazole (BTZ) monomer unit. We demonstrate that the BTZ unit allows for additional solubilizing side-chains on the co-monomer and show that the introduction of a linear side-chain on the DPP-unit leads to an increase in thin-film order and charge-carrier mobility if a sufficiently solubilizing, branched, side chain is attached to the BTZ. We compare two different synthetic routes, direct arylation and Suzuki-polycondensation, by a direct comparison of polymers obtained via the two routes and show that direct arylation produces polymers with lower electrical performance which we attribute to a higher density of chain Furthermore we demonstrate that a polymer utilizing this design motif and synthesized via Suzuki-polycondensation $\left(\left(l-C_{18}\right)\right.$-DPP-(b-C $\left.\left.{ }_{17}\right)-B T Z\right)$ exhibits exceptionally high and near balanced average electron and hole mobilities $>2 \mathrm{~cm}^{2} \mathrm{~V}^{-1} \mathrm{~s}^{-1}$ which are among the highest, robustly extracted mobility values reported for DPP copolymers in a top-gate configuration to date. Our results demonstrate clearly that linear side chain substitution of the DPP unit together with co-monomers that allow for the use of sufficiently long or branched solubilizing side chains can be an attractive design motif for solution processable, high mobility DPP copolymers.

Received 13th April 2015

Accepted 7th August 2015

DOI: $10.1039 / \mathrm{c} 5 \mathrm{sc0} 01326 \mathrm{~g}$

www.rsc.org/chemicalscience

\section{Introduction}

Low-bandgap, donor-acceptor conjugated copolymers are being intensely researched for applications in organic field-effect-

${ }^{a}$ Cavendish Laboratory, University of Cambridge, JJ Thomson Avenue, Cambridge, CB3 oHE, UK. E-mail: hs220@cam.ac.uk

${ }^{b}$ Department of Polymer Science \& Engineering, Inha University, Incheon, 402-751, South Korea.E-mail: jkl36@inha.ac.kr

'Department of Energy Conversion and Storage, Technical University of Denmark, Frederiksborgvej 399, 4000 Roskilde, Denmark

${ }^{d}$ Department of Materials Engineering, Monash University, Clayton, Victoria 3800, Australia

${ }^{e}$ School of Chemistry, Bio21 Institute, University of Melbourne, Parkville, Victoria 3010, Australia

${ }^{f}$ Department of Chemistry and Centre for Plastic Electronics, Imperial College, London, SW7 2AZ, UK. E-mail: m.heeney@imperial.ac.uk

$\dagger$ Electronic supplementary information (ESI) available: Raw materials and solvents, used equipment, synthesis routes, additional TGA, GIWAXS and T-dependent mobility data. See DOI: 10.1039/c5sc01326g Additional data related to this publication is available at https:/www.repository.cam.ac.uk/

\$ These authors contributed equally to this work. M. G. designed and performed the device fabrication and polymer characterization experiments, analysed the experimental data and wrote the paper while S.-H. J. synthesized the polymers

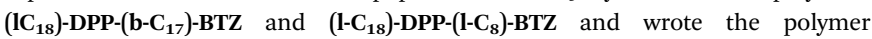
synthesis sections. transistors (OFETs) and solar cells. They have led to the development of polymer-based OFETs with high field-effect-mobilities exceeding $1 \mathrm{~cm}^{2} \mathrm{~V}^{-1} \mathrm{~s}^{-1}$ which outperform amorphous-Si (a-Si:H) thin film transistors and could enable new applications for OFETs, including logic circuit applications that require fast switching and current-driven flexible displays based on organic light-emitting diodes. ${ }^{1,2}$ For logic circuit applications a complementary circuit design based on p- and n-type FETs is beneficial to enable low-power consumption and achieve adequate noise margins for larger-scale integration. ${ }^{3}$ Polymers that are intrinsically ambipolar, i.e. capable of operating in either p-type or n-type operation mode, facilitate fabrication of such circuits, either using ambipolar or genuinely complementary logic configurations. In the latter case a single polymer semiconductor is used to realize both the p-type and the n-type devices, but the device configuration is engineered to suppress either electron or hole transport, respectively, through the use of appropriate sourcedrain contacts or gate dielectrics. ${ }^{4}$ Ambipolar polymer semiconductors have reached exceptionally high performances with $\mathrm{p}$ and n-type mobilities exceeding $2 \mathrm{~cm}^{2} \mathrm{Vs}^{-1}$ and simple ambipolar circuits, such as ring oscillators with oscillation frequencies of up to $380 \mathrm{kHz}$ and simple logic gates have been demonstrated. ${ }^{4-6}$

Some of the highest performing ambipolar donor-acceptor polymers known today are based on the diketopyrrolopyrrole 


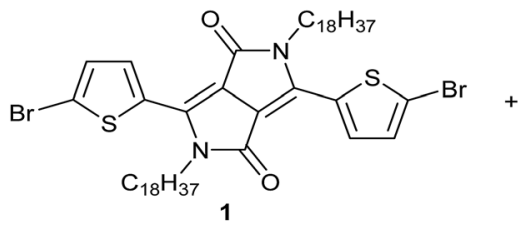

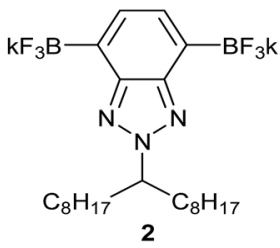

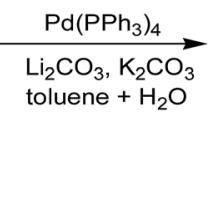

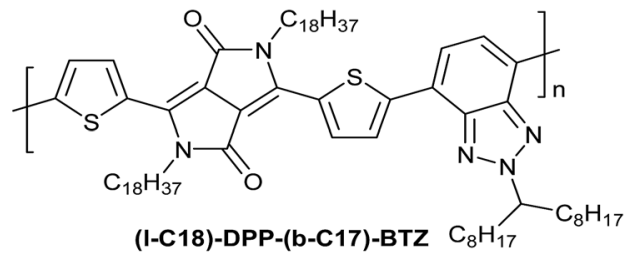

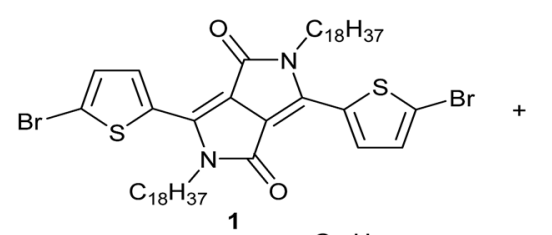

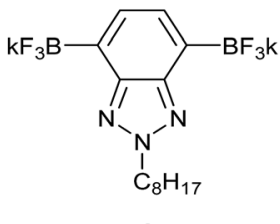

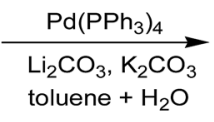

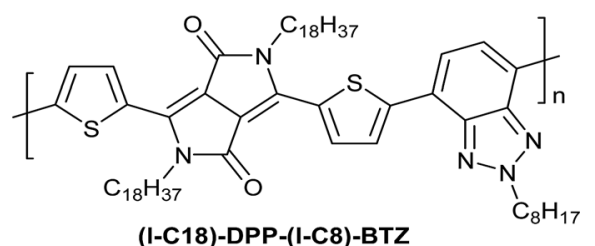

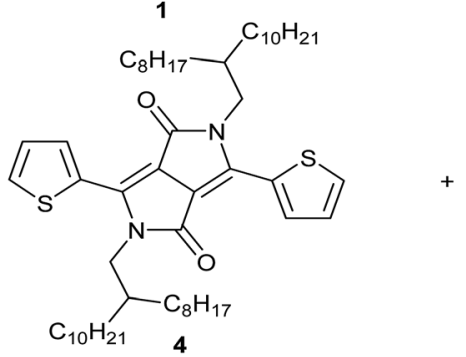
3

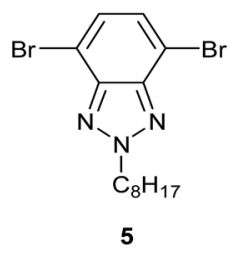

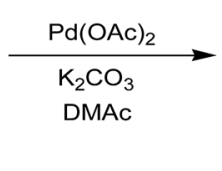

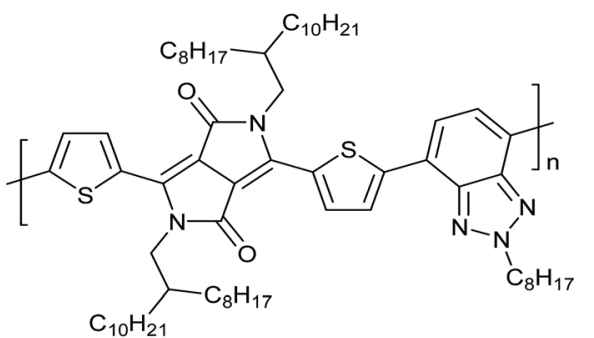
(b-C20)-DPP-(I-C8)-BTZ

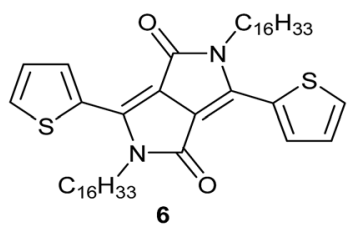

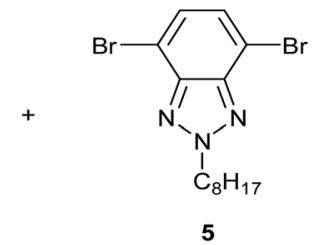
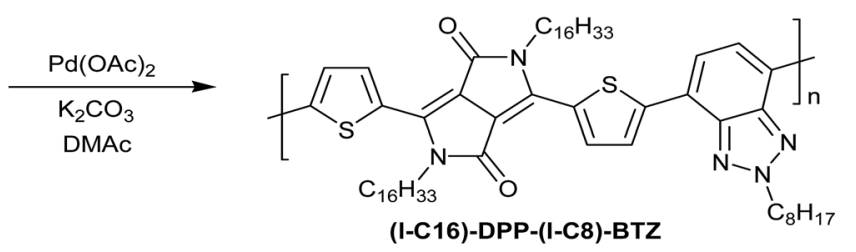

Scheme 1 Synthesis of the four DPP-benzotriazole copolymers investigated in this study.

(DPP) electron accepting unit. FET mobilities up to $12 \mathrm{~cm}^{2} \mathrm{~V}^{-1} \mathrm{~s}^{-1}$ for hole- and up to $6.3 \mathrm{~cm}^{2} \mathrm{~V}^{-1} \mathrm{~s}^{-1}$ for electron-transport have been claimed, ${ }^{2,6-8}$ although in these ambipolar materials there is ongoing debate about overestimation of mobility values, when mobilities are extracted from a narrow gate voltage range near the onset of hole accumulation, in which the hole concentration might be enhanced by residual electrons in the channel. The DPP unit is usually flanked by side units like phenylene, thiophene or selenophene. Units based on five-membered rings, such as thiophene or selenophene, tend to exhibit less backbone torsion and improved backbone co-planarity resulting in improved OFET performance compared to six-membered rings. ${ }^{9}$ Recently Nielsen and co-workers published a very extensive review comparing more than 80 different DPP containing polymers and summarizing the effects of different side chain substitution, co-monomers and processing conditions on FET performance. ${ }^{9}$ In most polymers containing the DPP unit, long, branched side-chains are attached to the DPP-core to endow the polymers with solution processability. It is generally understood that the side chain substitution exerts an important influence on the packing and backbone conformation of conjugated polymers in the solid state and may even dictate the ability of the polymer to form an ordered microstructure. ${ }^{10}$ Therefore choosing the right chemical type (e.g. alkyl, fluoroalkyl, ...), structure (e.g. linear or branched) and length of side-chains is an important part of conjugated polymer design and can lead to major differences in electrical performance of a semiconducting polymer. The deliberate choice of side-chains to enable good electrical performance of a conjugated polymer is referred to as side-chain engineering and has gained in importance in the last few years leading to an increasing number of publications on this topic. ${ }^{11}$ It has been found in a number of conjugated polymers that the introduction of branched instead of linear alkyl side-chains improves polymer solubility but is often detrimental for efficient charge-transport as e.g. shown by Osaka et al. and Guo et al. for two series of polymers in organic fieldeffect transistors. ${ }^{12}$ In their respective works both groups found decreased charge-carrier mobilities for polymers containing branched side-chains compared to the linear side-chain substituted variants and both authors attributed the decreased transistor performance to an increase in side-chain bulkiness of branched alkyl-chains preventing efficient charge-transport through the polymer film. However by examining a series of DPPbased polymers and varying the attachment density of a branched 2-octyldodecyl side-chain Zhang et al. found that branched sidechains can also improve charge-carrier mobility by facilitating a favourable polymer backbone alignment in the thin film. ${ }^{13}$ Based on these results other groups studied the effect of branched sidechains on polymer semiconductors by evaluating the influence of 
branching point position, as well as side chain type in various DPP-based polymers. ${ }^{14}$

However despite the increasing amount of publications on this topic no universal set of rules for the efficient design of side-chains in DPP-based conjugated polymers exists up to date. Therefore further studies on the effects of side-chain substitution on the electrical properties and morphology in a wider range of DPP copolymers are urgently needed. ${ }^{9}$ In this publication we therefore discuss the effects of linear and branched side-chains and their positioning on thin-film morphology and electrical performance in a novel dithienyl-DPP based polymer obtained by copolymerization with the weakly electron-deficient benzotriazole (BTZ) monomers (Scheme 1). The polymer allows for facile substitution with either linear or branched side chains on both the DPP-core and the BTZ co-monomer unit. To the best of our knowledge we report in this work the first DPP-based polymer FET with a mobility $>2 \mathrm{~cm}^{2} \mathrm{~V}^{-1} \mathrm{~s}^{-1}$ in which the DPP unit is substituted with a linear side chain. In fact, among the wide range of DPP polymers tested in our group under comparable conditions, for which mobilities were extracted in a robust manner and in a top-gate device architecture, this mobility is among the highest we have observed for this class of polymers. It is higher, for example, than the value we routinely observe for DPP-T-TT (ref. 6), one of the most widely studied and best performing DPP-copolymers.

\section{Experimental details}

\section{Materials}

$\mathrm{K}_{2} \mathrm{CO}_{3}, \mathrm{Li}_{2} \mathrm{CO}_{3}, \mathrm{Pd}\left(\mathrm{PPh}_{3}\right)_{4}, \mathrm{Pd}(\mathrm{OAc})_{2}$, phenylboronic acid, bromobenzene were purchased from Sigma-Aldrich or Acros and used as received. Toluene (anhydrous) and dimethylacetamide (DMAc) were received from Sigma-Aldrich and used without further purification. Monomers 2,5-di(2-octyldodecyl)-3,6-bis(thiophenyl)-1,4-diketopyrrolo[3,4-c]pyrrole and 2,5-dihexadecyl3,6-bis-(thiophenyl)-1,4-diketo-pyrrolo[3,4-c]pyrrole were prepared according to the literature procedures. ${ }^{15}$ The synthesis of the other monomers is given in the ESI. $\dagger$

\section{Polymer synthesis and characterisation}

(I-C 18$)$-DPP-(b-C 17$)-B T Z$ polymer prepared by a Suzuki polycondensation method. To a Schlenk tube were added dipotassium (b-C $\left.\mathrm{C}_{17}\right)-\mathrm{BTZ}$ bis(trifluoroborate) $2(0.200 \mathrm{~g}, 0.351 \mathrm{mmol})$, (l-C 18$)$-DPP dibromide $1(0.338 \mathrm{~g}, 0.351 \mathrm{mmol}), \operatorname{Pd}\left(\mathrm{PPh}_{3}\right)_{4}$ $(0.02 \mathrm{~g}, 0.018 \mathrm{mmol}), \mathrm{Li}_{2} \mathrm{CO}_{3}(0.400 \mathrm{~g}), \mathrm{K}_{2} \mathrm{CO}_{3}(0.400 \mathrm{~g})$ and $\mathrm{Bu}_{4} \mathrm{NBr}(0.011 \mathrm{~g}, 0.035 \mathrm{mmol}){ }^{16,17}$ The reaction mixture was then purged with $\mathrm{N}_{2}$. Toluene $\left(\mathrm{N}_{2}\right.$ bubbled, $\left.6 \mathrm{~cm}^{3}\right)$ was added to the Schlenk tube and the resulting mixture was stirred at $90{ }^{\circ} \mathrm{C}$ for $5 \mathrm{~min}$. Water $\left(\mathrm{N}_{2}\right.$ bubbled, $\left.4 \mathrm{~cm}^{3}\right)$ was added to the tube at $90{ }^{\circ} \mathrm{C}$. After the heterogeneous mixture was stirred for $4 \mathrm{~h}$ at $90{ }^{\circ} \mathrm{C}$, a solution of phenylboronic acid $(0.043 \mathrm{~g}, 0.35 \mathrm{mmol})$ dissolved in toluene $\left(\mathrm{N}_{2}\right.$ bubbled, $\left.2 \mathrm{~cm}^{3}\right)$ was added. The reaction mixture was stirred for another $1 \mathrm{~h}$ at $90{ }^{\circ} \mathrm{C}$. Bromobenzene $(0.11 \mathrm{~g}, 0.70 \mathrm{mmol})$ was added to the mixture. After the reaction mixture was stirred for further $2 \mathrm{~h}$, it was allowed to cool down to room temperature and precipitated into stirring $\mathrm{MeOH}$ $\left(200 \mathrm{~cm}^{3}\right)$. The precipitates were filtered, washed with $\mathrm{MeOH}$, and dried under reduced pressure. For purification of the polymer, the dried precipitate was dissolved in $\mathrm{CHCl}_{3}\left(300 \mathrm{~cm}^{3}\right)$ and a pipetteful amount of ammonium hydroxide was added to the solution. It was then passed through a short plug of silica gel with flushing with a copious amount of $\mathrm{CHCl}_{3}$ and concentrated under reduced pressure. The concentrated polymer solution was dropped into stirring $\mathrm{MeOH}\left(200 \mathrm{~cm}^{3}\right)$. The precipitated polymer was filtered, washed in a Soxhlet extraction apparatus with acetone for $48 \mathrm{~h}$ and dried under reduced pressure again to give the copolymer $\left(\mathbf{l}-\mathbf{C}_{\mathbf{1 8}}\right)$-DPP-(b- $\left.\mathbf{C}_{\mathbf{1 7}}\right)-\mathbf{B T Z}$ as a dark brown solid $(0.26 \mathrm{~g})$.

GPC (chlorobenzene, $80{ }^{\circ} \mathrm{C}$ ): $M_{\mathrm{n}}=63000 \mathrm{~g} \mathrm{~mol}^{-1}, M_{\mathrm{w}}=$ $204000 \mathrm{~g} \mathrm{~mol}^{-1}$, PDI $=3.2 . T_{\text {decomp }}$ (onset) $=390^{\circ} \mathrm{C}$. Found: $\mathrm{C}$, 75.6; $\mathrm{H}, 10.2 ; \mathrm{N}, 6.0 ; \mathrm{S}, 5.5 \% .\left(\mathrm{C}_{73} \mathrm{H}_{117} \mathrm{~N}_{5} \mathrm{~S}_{2} \mathrm{O}_{2}\right)_{n}$ requires: $\mathrm{C}, 75.5$; $\mathrm{H}, 10.2 ; \mathrm{N}, 6.0 ; \mathrm{S}, 5.5 \%$.

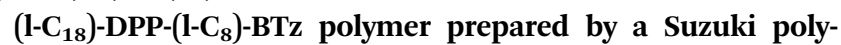
merisation method. Following the procedure above $\left(1-\mathrm{C}_{18}\right)$-DPP dibromide 1 (0.217 g, $0.226 \mathrm{mmol})$, dipotassium $\left(\mathrm{l}-\mathrm{C}_{8}\right)-\mathrm{BTZ}$ bis(trifluoroborate) $3(0.100 \mathrm{~g}, 0.226 \mathrm{mmol}), \mathrm{Pd}\left(\mathrm{PPh}_{3}\right)_{4}(0.013 \mathrm{~g}$, $0.011 \mathrm{mmol}), \mathrm{Li}_{2} \mathrm{CO}_{3}(0.200 \mathrm{~g}), \mathrm{K}_{2} \mathrm{CO}_{3}(0.200 \mathrm{~g}), \mathrm{Bu}_{4} \mathrm{NBr}$ $(0.0073 \mathrm{~g}, 0.023 \mathrm{mmol})$ in toluene $\left(3 \mathrm{~cm}^{3}\right)$ were reacted at $90{ }^{\circ} \mathrm{C}$ for $5 \mathrm{~min}$, after which water $\left(2 \mathrm{~cm}^{3}\right)$ was added. Following reaction for $4 \mathrm{~h}$, the mixture was endcapped as above using degassed solutions of phenylboronic acid $(0.028 \mathrm{~g}, 0.23 \mathrm{mmol})$ in toluene $\left(1 \mathrm{~cm}^{3}\right)$ and then bromobenzene $(0.071 \mathrm{~g}$, $0.45 \mathrm{mmol}$ ). The polymer as isolated as above to give the poly-

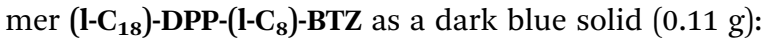

GPC (chlorobenzene, $80{ }^{\circ} \mathrm{C}$ ): $M_{\mathrm{n}}=11000 \mathrm{~g} \mathrm{~mol}{ }^{-1}, M_{\mathrm{w}}=$ $55000 \mathrm{~g} \mathrm{~mol}^{-1}$, PDI $=4.0 . T_{\text {decomp. }}$ (onset) $=413^{\circ} \mathrm{C}$. Found: $\mathrm{C}$, $72.7 ; \mathrm{H}, 9.4 ; \mathrm{N}, 6.5 ; \mathrm{S}, 6.1 \% .\left(\mathrm{C}_{64} \mathrm{H}_{99} \mathrm{~N}_{5} \mathrm{~S}_{2} \mathrm{O}_{2}\right)_{n}$ requires: $\mathrm{C}, 74.3$; $\mathrm{H}, 9.6 ; \mathrm{N}, 6.8 ; \mathrm{S}, 6.2 \%$.

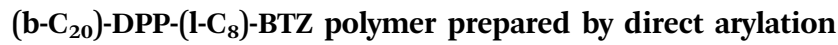
method. A mixture of $\left(\mathrm{b}-\mathrm{C}_{20}\right)$-DPP $4(0.146 \mathrm{~g}, 0.170 \mathrm{mmol}),\left(\mathrm{l}-\mathrm{C}_{8}\right)$ BTZ dibromide $5(0.0661 \mathrm{~g}, 0.170 \mathrm{mmol}), \mathrm{Pd}(\mathrm{OAc})_{2}(0.0016 \mathrm{~g}$, $0.007 \mathrm{mmol})$, and $\mathrm{K}_{2} \mathrm{CO}_{3}(0.0345 \mathrm{~g}, 0.25 \mathrm{mmol})$ in anhydrous DMAc $\left(5 \mathrm{~cm}^{3}\right)$ was purged with Ar stream and stirred for $72 \mathrm{~h}$ at $110{ }^{\circ} \mathrm{C}$ under Ar. $^{18}$ After cooling to room temperature, the reaction mixture was precipitated into acidic $\mathrm{MeOH}$ and stirred for another $30 \mathrm{~min}$. The resulting precipitates were filtered, washed with $\mathrm{MeOH}$ and purified by Soxhlet extraction with $\mathrm{MeOH}$, acetone and hexane before collecting the higher molecular weight $\mathrm{CHCl}_{3}$ fraction. To the $\mathrm{CHCl}_{3}$ extract was added an aqueous solution of sodium diethyldithiocarbamate (ca. $1 \mathrm{~g}$ per $100 \mathrm{~cm}^{3}$ ) and the mixture was heated to $60{ }^{\circ} \mathrm{C}$ with vigorous stirring for $2 \mathrm{~h}$. After cooling to room temperature, the layers were separated and the organic fraction was washed with water $\left(2 \times 150 \mathrm{~cm}^{3}\right)$, and concentrated under reduced pressure. The resulting residue was dissolved in a minimum amount of $\mathrm{CHCl}_{3}$ and added dropwise to a vigorously stirred $\mathrm{MeOH}$ $\left(250 \mathrm{~cm}^{3}\right)$. The precipitates were filtered using a $0.45 \mu \mathrm{m}$ PTFE filter and dried under reduced pressure to afford the desired polymer. A final purification by preparative GPC afforded the polymer as a dark powder $(0.083 \mathrm{~g}, 42 \%)$.

GPC (chlorobenzene, $80{ }^{\circ} \mathrm{C}$ ): $M_{\mathrm{n}}=93000 \mathrm{~g} \mathrm{~mol}^{-1} M_{\mathrm{w}}=$ $142000 \mathrm{~g} \mathrm{~mol}^{-1}$, PDI $=1.5$. Found: C, 74.1; H, 9.4; N, 6.1\%. $\left(\mathrm{C}_{68} \mathrm{H}_{107} \mathrm{~N}_{5} \mathrm{~S}_{2} \mathrm{O}_{2}\right)_{n}$ requires: C, 74.9; H, 9.9; N, 6.4\%. 
(1-C 16$)$-DPP-(1-C 8 )-BTZ prepared by direct arylation method. Using a similar procedure to above, 4,7-dibromo-2-octyl-2,1,3benzotriazole (66.1 mg, $0.17 \mathrm{mmol}$ ), 2,5-dihexadecyl-3,6-bis-(thiophenyl)-1,4-diketopyrrolo[3,4-c]pyrrole (127.4 mg, $0.17 \mathrm{mmol}$ ), $\mathrm{Pd}(\mathrm{OAc})_{2}(1.6 \mathrm{mg}, 0.007 \mathrm{mmol})$, and $\mathrm{K}_{2} \mathrm{CO}_{3}(34.5 \mathrm{mg}, 0.25 \mathrm{mmol})$ in anhydrous dimethylacetamide DMAc $\left(5 \mathrm{~cm}^{3}\right)$ were reacted for $72 \mathrm{~h}$ at $110{ }^{\circ} \mathrm{C}$. Following purification as above l-PDPPBTz was isolated as a dark powder. Yield: $77 \mathrm{mg}, 47 \%$.

GPC (chlorobenzene, $80{ }^{\circ} \mathrm{C}$ ): $M_{\mathrm{n}}=27600 \mathrm{~g} \mathrm{~mol}^{-1} ; M_{\mathrm{w}}=$ $54800 \mathrm{~g} \mathrm{~mol}^{-1}$; PDI = 2.0. Found: C, 72.9; H, 8.7; N, 6.7\%. $\left(\mathrm{C}_{60} \mathrm{H}_{89} \mathrm{~N}_{5} \mathrm{O}_{2} \mathrm{~S}_{2}\right)_{n}$ requires: $\mathrm{C}, 73.8 ; \mathrm{H}, 9.2 ; \mathrm{N}, 7.2 \%$.

\section{Device fabrication and characterisation}

Bottom-contact top-gate field-effect transistors were fabricated with poly(methyl methacrylate) (PMMA) as polymer gate dielectric. $20 \mathrm{~nm}$ Au source and drain contacts with a $4 \mathrm{~nm} \mathrm{Cr}$ adhesion layer were constructed photolithographically on Corning 1737 alkali-free borosilicate glass substrates. The prestructured substrates were then transferred into a nitrogen glovebox for all subsequent processing steps. All polymer semiconductor films with the exception of (1-C $\mathbf{1 8})-\mathbf{D P P}-\left(\mathbf{l}-\mathbf{C}_{\mathbf{8}}\right)$ BTZ were deposited on top of the electrodes by spin-coating from a $9 \mathrm{mg} \mathrm{mL^{-1 }}$ solution in anhydrous chlorobenzene

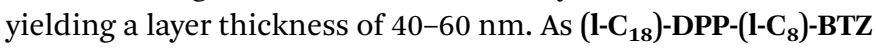
showed a very low solubility in chlorobenzene it was spin-coated from 1,2-dichlorobenzene using the same parameters as for the other polymers and yielding a comparable polymer film thickness. Afterwards the polymer films were annealed at the designated temperature for one hour before cooling to room temperature. The PMMA polymer gate dielectric film (from Polymer Source, electronic-grade, syndiotactic PMMA) was spincoated from a $50 \mathrm{mg} \mathrm{mL}^{-1}$ solution in anhydrous $n$-butylacetate and subsequently annealed at $90{ }^{\circ} \mathrm{C}$ for 30 minutes resulting in a $500 \mathrm{~nm}$ thick dielectric layer. Finally $\mathrm{Au}$ gate electrodes were evaporated thermally through a shadow mask to complete the devices. Due to the evaporator being located in ambient atmosphere the samples were taken out of the glovebox and mounted in the evaporator in ambient air prior to evaporation.

Electrical characterization of all devices was performed with an Agilent 4155B Semiconductor Parameter Analyzer inside a nitrogen glovebox. Saturated field-effect mobilities $\left(\mu_{\text {sat }}\right)$ were determined from the slope of $I_{\mathrm{D}}{ }^{1 / 2}$ over $V_{\mathrm{G}}$ in the last $10 \mathrm{~V}$ of the transfer curves $\left(\mu_{\mathrm{h}}: V_{\mathrm{G}}=-50\right.$ to $-60 \mathrm{~V}, \mu_{\mathrm{e}}: V_{\mathrm{G}}=50$ to $\left.60 \mathrm{~V}\right)$ by using the following equation:

$$
\mu_{\mathrm{sat}}=\left(\mathrm{d} I_{\mathrm{D}}^{1 / 2} / \mathrm{d} V_{\mathrm{G}}\right)^{2} 2 L /\left(W C_{\text {ins }}\right)
$$

with $L$ and $W$ being the transistor channel length and width and $C_{\text {ins }}$ being the gate dielectric capacitance. Due to a non-negligible gate-voltage dependency of charge-carrier mobilities, extracted mobility values were slightly lower if a larger gatevoltage region was used to determine mobility. However even if almost the whole transistor operating range $\left(\right.$ e.g. for $\mu_{\mathrm{h}}: V_{\mathrm{G}}=$ -60 to $-30 \mathrm{~V}$ ) was used for mobility extraction, extracted mobilities were still $70-90 \%$ of the values extracted in the last $10 \mathrm{~V}$ of the transfer curve and ranged up to $2 \mathrm{~cm}^{2} \mathrm{~V}^{-1} \mathrm{~s}^{-1}$ for the best devices. This shows that the used method for mobility extraction is robust and does not yield unrealistically high mobility values. For measurements at low temperatures a Desert Cryogenics low temperature probe station was used. Measurements at low temperatures were carried out by cooling the devices to low temperatures and reheating them afterwards. Measurement points were taken in both directions (during cooling and heating cycle).

\section{Thin-film structural characterization}

Synchrotron-based grazing-incidence wide-angle $\mathrm{X}$-ray scat-

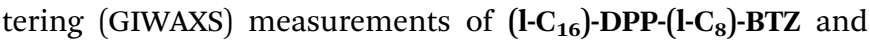

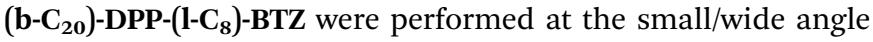
$\mathrm{X}$-ray scattering beamline at the Australian Synchrotron. ${ }^{19}$ $14 \mathrm{keV}$ photons were used with the 2D diffraction patterns recorded on an MAR-165 CCD detector. A grazing incidence angle of $0.1^{\circ}$, close to the films critical angle, was employed causing the X-rays to penetrate the entire film and therefore probe the films bulk structure. The sample-to-detector distance was calibrated using a silver behenate standard. Data acquisition times of $60 \mathrm{~s}$ were used with no evidence found for beam damage when comparing data taken at shorter and longer acquisition times. GIWAXS data were analysed using the software SAXS15ID version 3299. X-ray diffraction data are expressed as a function of the scattering vector $\boldsymbol{Q}$ that has a magnitude of $4 \pi / \lambda \sin \theta$, where $\theta$ is half the scattering angle and $\lambda$ is the wavelength of the incident radiation.

Additional GIWAXS measurements of (1-C $\left.\mathbf{C}_{\mathbf{1 8}}\right)$-DPP-(b-C $\left.\mathbf{C}_{\mathbf{1 7}}\right)$ -

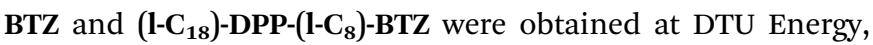
Risø Campus using $\mathrm{Cu} \mathrm{K}_{\alpha}$ radiation $(\lambda=1.542 \AA)$, generated by a rotating anode and focused using a $1 \mathrm{D}$ multilayer. ${ }^{20}$ The data were recorded on photostimulable image plates at a distance of $121 \mathrm{~mm}$ from the sample. A grazing incidence angle of $0.18^{\circ}$ was employed, just below the critical angle of the Si wafer substrates, thus maximizing the diffracted signal from the polymer film. GIWAXS data were analysed using the software SIMDIFFRACTION. ${ }^{21}$

\section{Results and discussion}

\section{Polymer design and synthesis}

Co-polymerisation of the DPP-unit with another electron-deficient co-monomer has been explored previously to control the optical and electronic properties of such polymers. Strong acceptor units like benzothiadiazole (BT) or benzobisthiadiazole (BBT) have been used and have led to high mobility ambipolar polymer semiconductors with very low band gaps $\left(E_{\mathrm{g}}<0.7 \mathrm{eV}\right)$ and high ambipolar charge-carrier mobilities slightly exceeding $1 \mathrm{~cm}^{2} \mathrm{~V}^{-1} \mathrm{~s}^{-1} \cdot{ }^{22}$ Despite their impressive performance, DPP polymers containing non-alkylated co-monomers are often poorly soluble even in chlorinated solvents and their solutions form gels when stored at room temperature. This constitutes important issues for their practical use. In this publication we investigate benzotriazole (BTZ) as an attractive alternative, weakly electron-deficient co-monomer unit. Compared to co-monomers like BT or BBT, BTZ offers 
additional solubility due to the possibility of alkylating the middle nitrogen atom. This allows us to explore the use of linear - as opposed to standard branched - side chains on the main DPP unit whilst maintaining a sufficient level of solubility and therefore enables the evaluation of the influence of side-chain type and positioning on FET performance in DPP-polymers.

We therefore synthesized four different modifications of a dithienyl-DPP-benzotriazole polymer with different linear and branched side chains on both the main DPP-core and the benzotriazole unit (see Scheme 1 for polymer chemical structures) to evaluate the influence of side-chain length, type and positioning on electrical performance. Despite the previous use of benzotriazole containing copolymers in organic photovoltaics and OFETs, to our knowledge this is the first demonstration of high performing OFETs based on DPP copolymers with a benzotriazole co-monomer. ${ }^{23}$

In order to access the four DPP-based polymers, we had to adopt two different synthetic protocols based on Pd catalysed cross-coupling, namely Suzuki polycondensation and direct arylation polymerisation (DARP). The first two polymers, $\left(\mathbf{1}-\mathbf{C}_{\mathbf{1 8}}\right)$ -

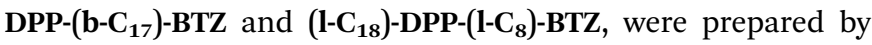
the Suzuki polycondensation reactions in which dipotassium 2-alkylbenzotriazolyl bis(trifluoroborate)s $\mathbf{2}$ and $\mathbf{3}$ were employed as stable alternatives to the boronic acid or boron ester of 2-alkylbenzotriazole. ${ }^{16}$ An interesting point in the synthesis of dipotassium ( $\left.1-\mathrm{C}_{8}\right)$-BTZ bis(trifluoroborate) 3 is the difficulty in transforming dibromo-2-octylbenzotriazole into the latter through a sequence of lithiation employing ${ }^{t} \mathrm{BuLi}$, substitution reaction with isopropoxydioxaborolane and fluorination using an aqueous $\mathrm{KHF}_{2}$ solution. ${ }^{16}$ Only a tiny amount of impure monomer 3 was recovered after recrystallization, which totally differs from the case of dipotassium $\left(\mathrm{b}-\mathrm{C}_{17}\right)$-BTZ bis(trifluoroborate) 2. It seems that the dibromo-2-octylbenzotriazole decomposes in the presence of ${ }^{t} \mathrm{BuLi}$. Several consecutive, failures with modified reaction conditions made us look at alternative protocols, one of which was the Suzuki-Miyaura borylation using bis(pinacolato)diboron and $\mathrm{Pd}(\mathrm{dppf}) \mathrm{Cl}_{2}$ catalyst. $^{24} \mathrm{~A}$ sequence of borylation, conversion to the bis(trifluoroborate) 3 and crystallization from a mixture of acetonitrile and water provided the monomer 3 suitable for the synthesis of (1-C $\mathbf{1 8})$-DPP-(1-C $\mathbf{8})$-BTZ. Detailed synthetic procedures of relevant monomers are described in the ESI. $\dagger$

Another important point in the synthesis of polymers is the previously reported modification of the polymerisation conditions. ${ }^{16}$ For the polymerisation of dibromobenzothiadiazole and dipotassium (b-C 17 )-BTZ bis(trifluoroborate) 2, successful Suzuki polycondensations were possible employing a mixture of $\mathrm{Et}_{4} \mathrm{NOH}$ and $\mathrm{LiOH}$. However, the same reagents were not effective for promoting condensation reactions with $\left(1-\mathrm{C}_{18}\right)$-DPP dibromide 1 . Only a small amount of precipitate was recovered by filtration and the solid had low molecular weight. We therefore carefully looked into the polymerisation conditions, and came to suspect an incompatibility of the hydrolysable amide functional groups in the DPP unit with the strongly basic conditions induced by $\mathrm{Et}_{4} \mathrm{NOH}$ and $\mathrm{LiOH}$. Switching to a less basic reagent mixture of $\mathrm{K}_{2} \mathrm{CO}_{3}$ and $\mathrm{Li}_{2} \mathrm{CO}_{3}$ was thus regarded as a viable option, which allowed us to acquire the desired

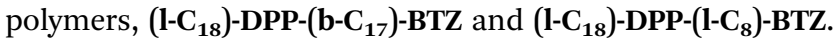

The challenges in obtaining the boronic acid derivatives of monomer 3 discussed above prompted the investigation of alternative polymerization routes. In particular we were interested in methods which could utilize the readily available dibromo-2-octylbenzotriazole monomer 5 directly. Direct arylation polymerization (DARP) was of particular interest since organometallic coupling groups do not need to be introduced onto the DPP or BTz monomers, potentially facilitating synthesis and scale-up. ${ }^{25}$ However the selectivity of the polymerization when there are multiple aromatic $\mathrm{C}-\mathrm{H}$ bonds present is a concern in some cases, and there have also been reports of significant homo-coupling of both monomers depending on the reaction conditions. ${ }^{\mathbf{2 6 , 2 7}}$ This is a potentially significant limitation, since such defects would be chemically bound to the polymer, and could not be removed. However it is challenging to directly identify such defects in conjugated polymers, particularly with polymers which show strong aggregation in solution such as DPP based materials, due to both the low number of potential defects present and also the poor resolution of NMR based techniques for such aggregated materials. Here by preparing similar straight chain polymers,

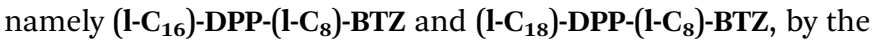
DARP and Suzuki routes we have an indirect method to compare the two polymerization routes, by investigating the thin film morphology and device performance of the two polymers.

Thus a co-polymer containing all linear sidechains, $\left(\mathbf{l}-\mathbf{C}_{\mathbf{1 6}}\right)$ -

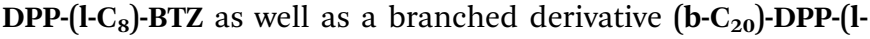
$\mathbf{C}_{8}$ )-BTZ in which the branching sidechain was on the DPP unit were prepared. Both polymers were obtained using the phosphine free conditions described by Scherf and co-workers utilizing $\mathrm{Pd}(\mathrm{OAc})_{2}$ as a catalyst with potassium carbonate in DMAc. $^{28}$ Following purification by solvent extraction and preparative GPC, the two polymers were isolated in yields of $\sim 40 \%$.

The molecular weights of all four polymers were measured by gel permeation chromatography in chlorobenzene at $80^{\circ} \mathrm{C}$ against polystyrene standards, and the results are shown in Table 1. All four polymers show relatively high average molecular weights $\left(M_{\mathrm{w}}\right)$ with broadly comparable values obtained for both polymerization methods. It is notable that both sets of branched polymers have considerably higher molecular weight than the linear polymers made by the same polymerization chemistry, which we ascribe to the improved solubility of the branched sidechain polymers. This helps to keep the growing polymer chain in solution during the polymerization process. Attempts to characterize the polymers by ${ }^{1} \mathrm{H}$ NMR were not very informative due to the broad, poorly resolved peaks observed. This is a common issue for relatively rigid low band gap polymers which show pronounced aggregation in solution.

\section{Polymer properties}

The UV-VIS absorption characteristics of the different DPP-BTZ variations in thin-film and in chlorobenzene solution are shown 
Table 1 Properties of the synthesized polymers. Number and weight average molecular weight $\left(M_{n} / M_{w}\right)$, polydispersity index (PDI), maximum absorption wavelength in chlorobenzene solution and in thin-films $\left(\lambda_{\text {max,solution }} / \lambda_{\text {max, film }}\right)$ estimated optical band gap $\left(E_{\mathrm{g}}\right)$ and average extracted hole and electron mobility in saturation at optimized film annealing temperature $\left(\mu_{\mathrm{h}} / \mu_{\mathrm{e}}\right)$

\begin{tabular}{|c|c|c|c|c|c|c|c|c|}
\hline & $M_{\mathrm{n}} / M_{\mathrm{w}}{ }^{a}[\mathrm{kDa}]$ & PDI & $\lambda_{\max , \text { solution }}[\mathrm{nm}]$ & $\lambda_{\text {max }, \text { film }}[\mathrm{nm}]$ & $E_{\mathrm{g}}{ }^{b}[\mathrm{eV}]$ & $\begin{array}{l}\mu_{\mathrm{h}} \\
{\left[\mathrm{cm}^{2} \mathrm{~V}^{-1} \mathrm{~s}^{-1}\right]}\end{array}$ & $\mu_{\mathrm{e}}\left[\mathrm{cm}^{2} \mathrm{~V}^{-1} \mathrm{~s}^{-1}\right]$ & $I_{\mathrm{on}} / I_{\mathrm{off}}$ \\
\hline$\left(1-C_{18}\right)-D P P-\left(b-C_{17}\right)-B T Z$ & $63 / 204$ & 3.2 & 858 & 875 & 1.25 & 2.4 & 1.5 & $>10^{7}$ \\
\hline$\left(1-C_{18}\right)-D P P-\left(1-C_{8}\right)-B T Z$ & $11 / 55$ & 4.0 & 764 & 860 & 1.32 & 0.48 & 0.31 & $>10^{5}$ \\
\hline$\left(\mathrm{b}-\mathrm{C}_{20}\right)-\mathrm{DPP}-\left(\mathrm{l}-\mathrm{C}_{8}\right)-\mathrm{BTZ}$ & $93 / 142$ & 1.5 & 836 & 840 & 1.30 & 0.011 & 0.018 & $>10^{4}$ \\
\hline
\end{tabular}

${ }^{a}$ Determined by GPC. ${ }^{b}$ Determined from onset of optical absorption.

in Fig. 1. The band gaps of all polymers described in this work are estimated from the onset of the low energy absorption peak in the solid state and are shown together with other physical data in Table 1.

All four polymers exhibit a double peak in their absorption spectra commonly observed in many DPP based polymers and a broad absorption band ranging from 500 up to $1040 \mathrm{~nm} .^{2,4}$

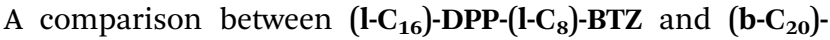

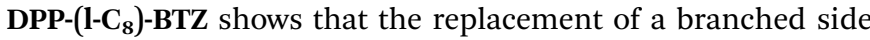
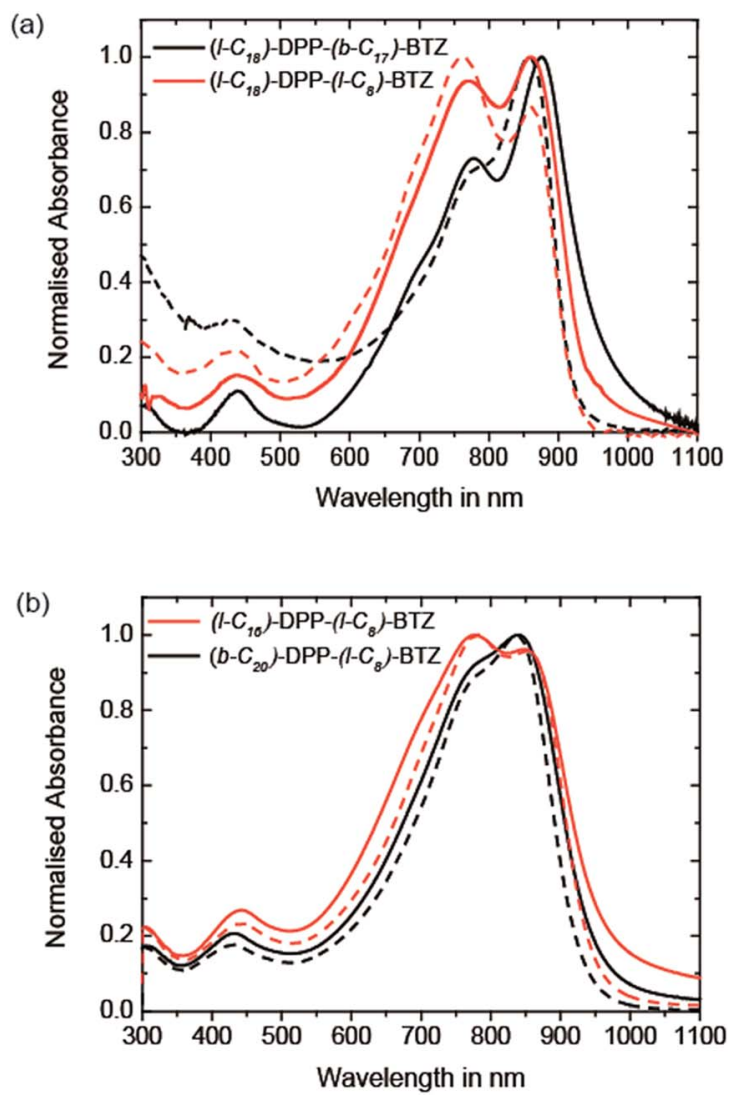

Fig. 1 (a) UV-VIS absorption spectra of (l-C 18$)$-DPP- $\left(b-C_{17}\right)-B T Z$ (black) and (l-C $\left.\mathrm{C}_{18}\right)$-DPP-(l-C $\left.\mathrm{C}_{8}\right)$-BTZ (red) measured in chlorobenzene solution (dashed lines) and thin-films (solid lines). (b) UV-VIS absorption spectra of $\left(\mathrm{l}-\mathrm{C}_{16}\right)-\mathrm{DPP}-\left(\mathrm{l}-\mathrm{C}_{8}\right)-\mathrm{BTZ}(\mathrm{red})$ and $\left(\mathrm{b}-\mathrm{C}_{20}\right)-\mathrm{DPP}-\left(\mathrm{l}-\mathrm{C}_{8}\right)-$ BTZ (black) measured in chlorobenzene solution (dashed lines) and thin-films (solid lines). chain at the DPP unit with a longer linear side chain results in a major change in absorption behaviour. The replacement leads to an increase in intensity of the $0-1$ absorption peak at $777 \mathrm{~nm}$ while the 0-0 absorption peak shifts from 840 to $849 \mathrm{~nm}$ and decreases in intensity. It also leads to a slightly lower band gap

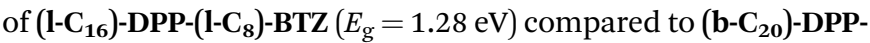
$\left(\mathbf{l}-\mathrm{C}_{8}\right)-\mathbf{B T Z}\left(E_{\mathrm{g}}=1.30 \mathrm{eV}\right)$ which is indicative of a longer conjugation length. In both polymers the solution and thin film spectra look alike suggesting similar polymer conformations in the solution and solid states.

Comparing the two linear sidechain polymers made by

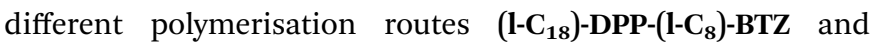

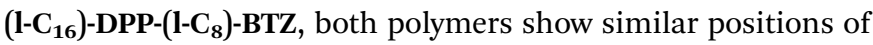
the two main peaks in solution (861 and $761 \mathrm{~nm}$ ) and in thinfilms (860 and $770 \mathrm{~nm}$ ). However, we detect a change in the

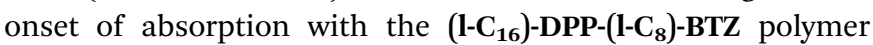
prepared by direct arylation exhibiting a less well defined transition and a smaller band gap of $1.28 \mathrm{eV}$ compared to

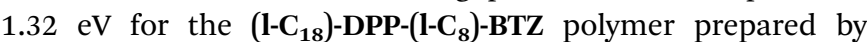
Suzuki polycondensation. These changes are similar to those recently identified by Janssen $e t$ al. and are most likely the result of homo-coupling defects during the DARP polymerization. ${ }^{29}$ Such homo couplings would likely act as traps.

The most interesting behaviour is exhibited by (1-C $\left.\mathbf{C}_{\mathbf{1 8}}\right)$-DPP(b-C $\left.{ }_{17}\right)$-BTZ, which has a branched side-chain attached to the BTZ unit together with a slightly longer linear side-chain on the DPP unit. It exhibits the best resolved fine structure of the absorption bands and the largest 0-0/0-1 peak ratio indicating a small reorganisation energy. Together with the small band gap value of $1.25 \mathrm{eV}$ and a considerable red shift of the main absorption maximum from 858 to $875 \mathrm{~nm}$ upon thin-film formation this suggests that the most planar, ordered backbone conformation and the longest conjugation length among the four polymers is present in $\left(\mathbf{l}-\mathbf{C}_{\mathbf{1 8}}\right)$-DPP-(b-C $\left.\mathbf{C}_{\mathbf{1 7}}\right)-\mathbf{B T Z}$. This is fully consistent with the FET performance reported below.

\section{Thin-film microstructure}

The 2D GIWAXS patterns of films of all four polymers annealed at temperatures to achieve optimum charge-transport are pre-

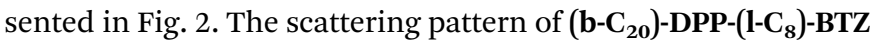
(see Fig. 2c) is essentially featureless indicating a lack of longrange periodic order and a near amorphous microstructure. The ring at $Q \sim 0.38 \AA^{-1}$ is due to scattering from a Kapton window 


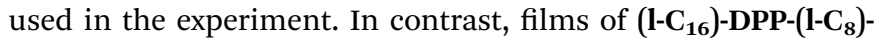
BTZ (see Fig. 2d) exhibit a series of (h00) scattering peaks oriented along $\boldsymbol{Q}_{z}$ corresponding to a semicrystalline microstructure with edge-on orientated lamellae. The (100) peak is located at $\boldsymbol{Q}_{z} \sim 0.27 \AA^{-1}$ corresponding to a lamellar stacking distance of $\sim 23.2 \AA$. A faint (010) stacking peak is observed at $\boldsymbol{Q}_{x y} \sim 1.75 \AA^{-1}$, corresponding to a $\pi-\pi$ stacking distance of

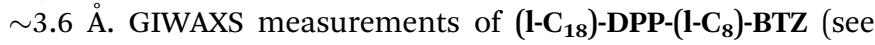
Fig. 3b) show a comparable semicrystalline thin-film structure

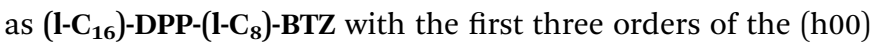
scattering peaks along the $\boldsymbol{Q}_{z}$-axis and a $\pi-\pi$ stacking peak at $Q_{x y} \sim 1.75 \AA^{-1}$. The position of the (100) peak at $\boldsymbol{Q}_{z} \sim 0.24 \AA^{-1}$ corresponds to a lamellar stacking distance of $\sim 25.7 \AA$. This slightly longer stacking distance is consistent with the difference in side-chain length between the two polymers. However

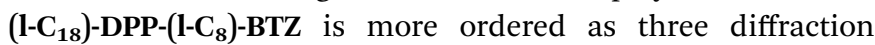
orders along $\boldsymbol{Q}_{z}$ are clearly visible compared to two peaks in $\left(\mathbf{I}-\mathbf{C}_{16}\right)-$ DPP-(I-C $\left.\mathbf{C}_{8}\right)-\mathbf{B T Z}$ with a weak indication of the $3^{\text {rd }}$ order peak. Furthermore, the pi-stacking peak is better defined for this polymer. The ring at $1.4 \AA^{-1}$ is due to packing of disordered side-chains.

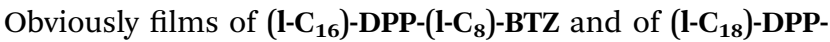
(1-C $\left.\mathbf{C}_{8}\right)$-BTZ which both incorporate a linear side-chain on the DPP-unit are more ordered than films of the (b-C $\left.\mathbf{C}_{20}\right)$-DPP-(1- $\left.\mathbf{C}_{8}\right)$ BTZ polymer with a branched side-chain on the DPP unit. This is not entirely unexpected and is consistent with results from Tamayo et al. who studied film-order in films formed of DPP molecules and observed a similar increase in film disorder when incorporating branched side-chains at the DPP-core. ${ }^{30}$ This is likely because linear side-chains on the DPP-unit do not
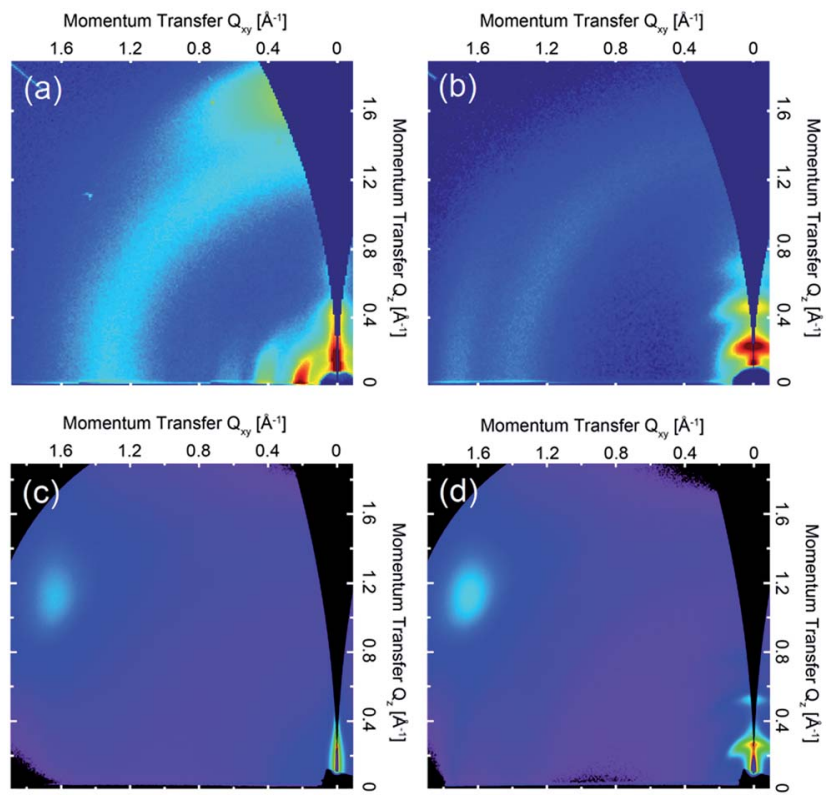

Fig. 2 2D GIWAXS patterns of (a) $\left(l-C_{18}\right)$-DPP- $\left(b-C_{17}\right)-B T Z$ film annealed at $110^{\circ} \mathrm{C}$, (b) $\left(\mathrm{l}-\mathrm{C}_{18}\right)$-DPP-(l-C 8$)-B T Z$ film annealed at $110^{\circ} \mathrm{C}$, (c) (b-C $\left.C_{20}\right)$-DPP-(l- $\left.C_{8}\right)-B T Z$ annealed at $200^{\circ} \mathrm{C}$ and (d) (l- $\left.C_{16}\right)$-DPP-(l$\mathrm{C}_{8}$ )-BTZ also annealed at $200^{\circ} \mathrm{C}$. Intensities are shown on a false color log scale.
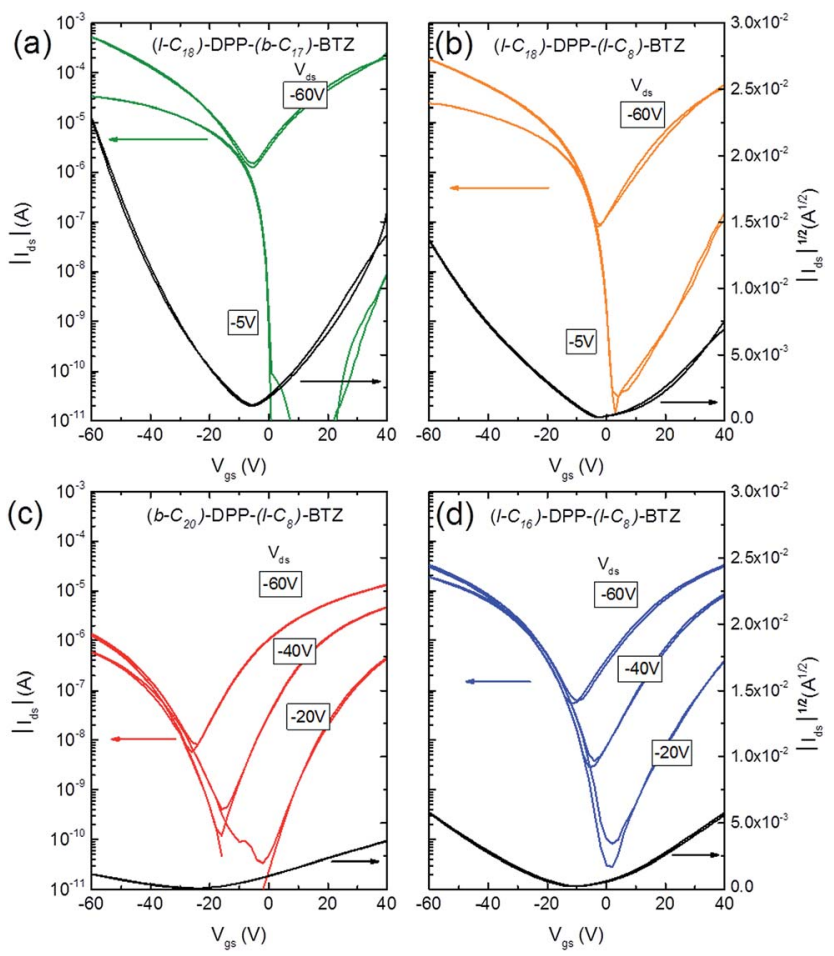

Fig. 3 Transfer characteristics of ambipolar transistors $(L=20 \mu \mathrm{m}$, $W=1000 \mu \mathrm{m})$ based on (a) (l-C 18$)$-DPP- $\left(b-C_{17}\right)$-BTZ annealed at

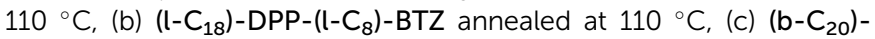
DPP-(l- $\left.\mathrm{C}_{8}\right)-\mathrm{BTZ}$ annealed at $200{ }^{\circ} \mathrm{C}$ and (d) (l- $\left.\mathrm{C}_{16}\right)-\mathrm{DPP}-\left(\mathrm{l}-\mathrm{C}_{8}\right)-\mathrm{BTZ}$ annealed at $200^{\circ} \mathrm{C}$.

interfere with aggregation as much as branched side-chains which provide a higher level of steric hindrance.

A different behaviour is observed when the branched sidechain is not incorporated on the DPP-unit but on the BTZ unit instead. As the GIWAXS data in Fig. 2a clearly shows, thin-films

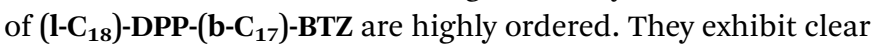
semicrystalline order but with a different texture as evidenced by the occurrence of the h00 peaks along the $\boldsymbol{Q}_{x y}$ direction, i.e. a face-on orientation with the $\pi$-stacking peak centred along the $\boldsymbol{Q}_{z}$ axis (surface normal). The 100 peak is located at $\boldsymbol{Q}_{x y} \sim$ $0.22 \AA^{-1}$, corresponding to a lamellar packing distance of $\sim 28.6 \AA$, and second and third order diffraction peaks at higher $Q_{x y}$ values. Peaks at the same positions are present in the out-ofplane direction as well but show reduced intensity compared to the in-plane peaks. This likely corresponds to a minority component of edge-on oriented crystallites. The weak ring at $q \sim 1.3 \AA^{-1}(\sim 4.8 \AA)$ is most likely caused by packing of disordered side-chains. These results indicate a distribution of faceon and edge-on oriented crystallites with a preferred face-on crystal orientation and a high overall degree of out-of-plane $\pi-\pi$ stacking with short $(\sim 3.7 \AA) \pi-\pi$ stacking distance.

Obviously the positioning of a long branched side-chain at the BTZ unit in comparison to a linear side-chain leads to a significant enhancement in thin-film order. This is especially

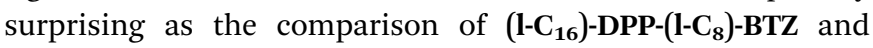

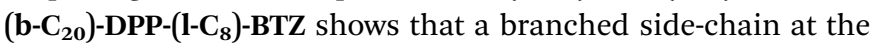
main DPP-core significantly hinders the formation of an 
ordered film in comparison to a linear side-chain. The strong tendency of the DPP-units to form aggregated structures is widely known and was already shown for oligothiophene DPP small molecules by Tamayo et al. who demonstrated that the DPP units stack in a coplanar fashion and form columnar stacks. ${ }^{30}$ It is not unreasonable to assume that the same stacking mechanism of DPP-units is present in DPP-containing polymers but can be hindered by bulky side-chains. Clearly, the addition of a branched side-chain at the BTZ unit not only improves solubility but also does not hinder stacking of DPP units which is evident from the high degree of $\pi-\pi$ stacking present in thin-films of (1-C $\left.\mathbf{C}_{\mathbf{1 8}}\right)$-DPP-(b-C $\left.\mathbf{1}_{\mathbf{1 7}}\right)$-BTZ. Furthermore it is interesting to note that a high degree of thin-film order is already present at low annealing temperatures of $110^{\circ} \mathrm{C}$ and can be further improved using higher annealing temperatures up to $300{ }^{\circ} \mathrm{C}$ (see Fig. S3†).

\section{Charge-transport and ambipolar transistors}

Fig. 3 shows representative transfer characteristics of bottomcontact top-gated field-effect transistors based on the four versions of DPP-BTZ polymer. As dielectric a $500 \mathrm{~nm}$ thick PMMA layer was used to prevent electron trapping at the semiconductor-dielectric interface and therefore enable n-type or ambipolar charge-transport., ${ }^{\mathbf{4 , 6}, 31}$ All transistors exhibit ambipolar transfer characteristics with low hysteresis and in the

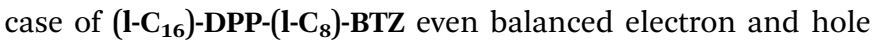
transport. The extracted saturated hole- and electron-mobilities of all four polymers as a function of temperature are shown in Fig. 4 .

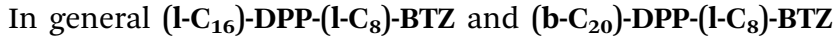
which were synthesized by direct arylation show lower transistor performances compared to (1-C $\mathbf{1 8})$-DPP-(b-C $\left.\mathbf{C}_{\mathbf{1 7}}\right)-\mathbf{B T Z}$ and

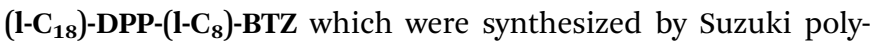
condensation. Both polymers exhibit a similar trend of higher annealing temperatures resulting in increased saturated fieldeffect mobilities.

The maximum extracted values for hole and electron mobilities were obtained after annealing at $300{ }^{\circ} \mathrm{C}$ and amount to 0.075 and $0.073 \mathrm{~cm}^{2} \mathrm{~V}^{-1} \mathrm{~s}^{-1}$ for $\left(\mathbf{1 - C _ { 1 6 }}\right)$-DPP-(1-C $\left.\mathbf{8}\right)-\mathbf{B T Z}$ and 0.011 and $0.018 \mathrm{~cm}^{2} \mathrm{~V}^{-1} \mathrm{~s}^{-1}$ for $\left(\mathbf{b}-\mathbf{C}_{20}\right)$-DPP-(1-C $\left.\mathbf{C}_{\mathbf{8}}\right)$-BTZ respectively. The almost seven-fold increase of charge carrier mobil-

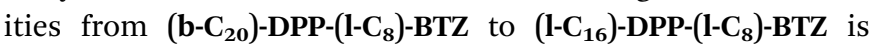
consistent with the increase in thin-film order as observed in the GIWAXS data. The linear side-chains used for (1-C $\left.\mathbf{1 6}_{\mathbf{1 6}}\right)$-DPP-(1$\mathbf{C}_{\mathbf{8}}$ )-BTZ lead to a better $\pi-\pi$ stacking and improved chargetransport.

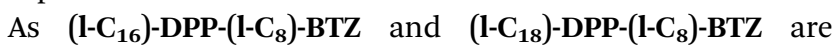
almost identical in terms of their chemical structure and mainly differ in terms of synthesis method (DARP vs. Suzuki polycondensation), molecular weight (see Table 1) and a small difference in side-chain length, it is interesting to draw a direct comparison between the transistor performance of the two

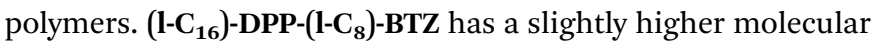

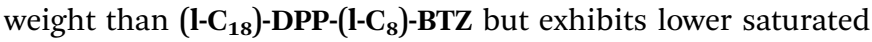
electron and hole mobilities of $0.075 \mathrm{~cm}^{2} \mathrm{~V}^{-1} \mathrm{~s}^{-1}$ and 0.073 $\mathrm{cm}^{2} \mathrm{~V}^{-1} \mathrm{~s}^{-1}$ compared to $0.48 \mathrm{~cm}^{2} \mathrm{~V}^{-1} \mathrm{~s}^{-1}$ and $0.31 \mathrm{~cm}^{2} \mathrm{~V}^{-1} \mathrm{~s}^{-1}$

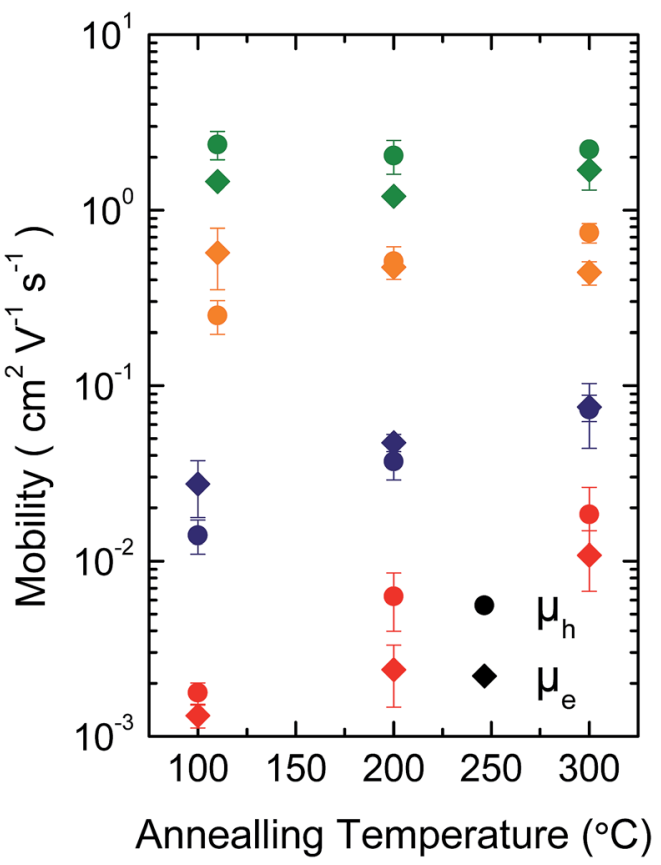

Fig. 4 Temperature dependence of saturated hole (circles) and electron (diamonds) mobility of (l- $\left.C_{18}\right)$-DPP- $\left(b-C_{17}\right)-B T Z$ (green),

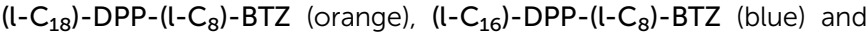
$\left(\mathrm{b}-\mathrm{C}_{20}\right)-\mathrm{DPP}-\left(\mathrm{l}-\mathrm{C}_{8}\right)-\mathrm{BTZ}$ (red).

for $\left(\mathbf{l}_{-\mathbf{C}_{\mathbf{1 8}}}\right)$-DPP-( $\left.\mathbf{l C}_{\mathbf{8}}\right)$-BTZ. Since in most polymer systems mobility is found to increase with molecular weight rather than decrease, the difference in device performance between these two polymers is most likely a reflection of the different synthesis routes used. The direct arylation method has been reported to incorporate homo-coupling defects into the chain, and such defects are expected to affect device performance adversely. ${ }^{27,29}$ Note that evidence for such homo-coupling defects was found in the UV-VIS spectra above. The increase in mobility is consistent with the higher degree of thin-film order as seen in the GIWAXS data.

As might have been anticipated from the high degree of microstructural order evident in GIWAXS, transistors based on (1-C $\left.\mathbf{C}_{\mathbf{1 8}}\right)$-DPP-(b-C $\left.\mathbf{1}_{\mathbf{1 7}}\right)$-BTZ show the highest FET performance (see Fig. 3a) with high $I_{\text {on }} / I_{\text {off }}$ ratios of $10^{6}$ to $10^{7}$ at low source-drain voltages $\left(V_{\mathrm{D}}=-5 \mathrm{~V}\right)$, low subthreshold swing values of 1.5 $\mathrm{V} \operatorname{dec}^{-1}$ and high average saturated hole mobilities of 2.4 $\mathrm{cm}^{2} \mathrm{~V}^{-1} \mathrm{~s}^{-1}$ with maximum values for hole mobilities ranging up to $2.8 \mathrm{~cm}^{2} \mathrm{~V}^{-1} \mathrm{~s}^{-1}$. Electron injection from the $\mathrm{Au}$ contacts

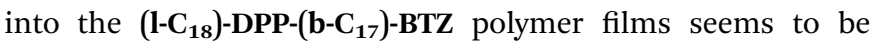
slightly hindered as evident from the lack of transfer curve symmetry, especially at low gate voltages $\left(V_{\mathrm{D}}=-5 \mathrm{~V}\right)$ and by the high relative voltage difference between drain and gate electrode needed to induce n-type transport. It was not possible to operate the transistors in the electron transport regime while using positive source-drain voltages because the high positive gate voltages needed to observe n-type transport using positive $V_{\mathrm{D}}$ usually lead to a breakdown of the polymer gate dielectric.

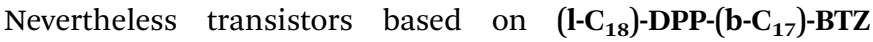
showed high average saturated electron mobilities around 
$1.5 \mathrm{~cm}^{2} \mathrm{~V}^{-1} \mathrm{~s}^{-1}$ with maximum electron mobilities ranging up to $2.4 \mathrm{~cm}^{2} \mathrm{~V}^{-1} \mathrm{~s}^{-1}$. The mobilities achieved with (1-C $\left.\mathbf{1 8}\right)$-DPP(b-C $\left.\mathbf{1}_{\mathbf{1 7}}\right)$-BTZ for either holes and electrons are amongst the highest values published for DPP-containing polymer semiconductors in top-gated structures. ${ }^{\mathbf{4 , 6 , 7 , 3 2}}$ Even though mobilities exceeding $3 \mathrm{~cm}^{2} \mathrm{~V}^{-1} \mathrm{~s}^{-1}$ have been published for devices using bottom-gated structures, these devices usually exhibit severe non-idealities in their device characteristics which manifest as kinks in the $I_{\mathrm{D}}{ }^{1 / 2}$ over $V_{\mathrm{G}}$ plot. These kinks lead to an unusual gate-voltage dependency of mobilities with very high mobilities in a small region at low $V_{\mathrm{G}}$ and much lower mobilities at higher $V_{\mathrm{G}}$ when the transistor is switched on completely. ${ }^{2,22,33}$ As pointed out in the experimental section our transistors do not show this behaviour and the extracted mobilities are valid in a large region of the transfer curve. As obvious from the transport data, the introduction of a branched side-chain at the BTZ unit as in (1-C $\left.\mathbf{C}_{\mathbf{1 8}}\right)$-DPP-(b-C $\left.\mathbf{C}_{\mathbf{1 7}}\right)$-BTZ improves charge-transport significantly compared to the linear side-chain in (1-C $\mathbf{1 8})$-DPP-(1-C $\left.\mathbf{C}_{\mathbf{8}}\right)$ BTZ. However it is still surprising that the introduction of a single branched side-chain on the BTZ unit leads to such a dramatic improvement in charge-carrier mobility compared to a linear side-chain. A possible explanation for this is the planarization of the polymer backbone by a more favourable sidechain arrangement leading to an improvement in thin-film order, a more planar backbone conformation with longer conjugation lengths and a reduced reorganization energy. This is consistent with the absorption spectrum of (1-C $\left.\mathbf{C}_{\mathbf{1 8}}\right)$-DPP(b-C $\mathbf{1 7}_{\mathbf{1 7}}$ )-BTZ showing a high 0-0/0-1 absorption peak intensity ratio which is indicative for a low reorganization energy and long conjugation lengths.

Low reorganization energies caused by different structural arrangement due to differences in side-chain packing in (1-C $\left.\mathbf{C}_{\mathbf{1 8}}\right)$ -

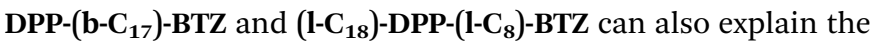
difference in annealing dependence of the saturation mobilities

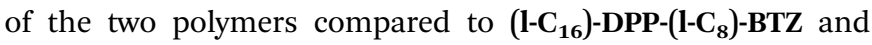

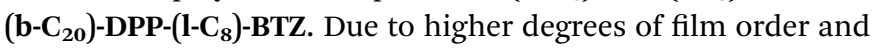
therefore low-reorganization energies already being present at

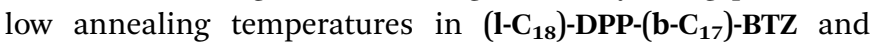

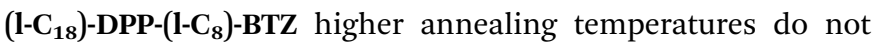
lead to large enhancements in charge-carrier mobility in these

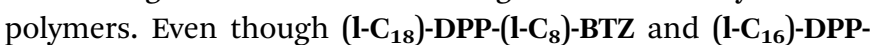
$\left(\mathbf{l}_{\mathbf{8}}\right)$-BTZ mainly differ in molecular weight, a small difference in side-chain length and the used synthesis method, the polymers do not show the same annealing behavior. If annealed at temperatures higher than $110{ }^{\circ} \mathbf{C}$ the hole mobility of $\left(\mathbf{1}-\mathbf{C}_{\mathbf{1 8}}\right)$ DPP-(1-C $\left.\mathbf{C}_{\mathbf{8}}\right)$-BTZ decreases while the electron mobility steadily increases up to annealing temperatures of $300{ }^{\circ} \mathbf{C}$. $\left(\mathbf{l}^{\left.-\mathbf{C}_{\mathbf{1 8}}\right)}\right.$-DPP(b-C $\left.\mathbf{C}_{\mathbf{1 7}}\right)$-BTZ essentially shows the same annealing behavior as

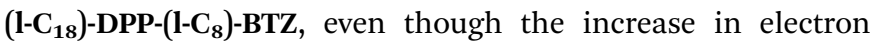

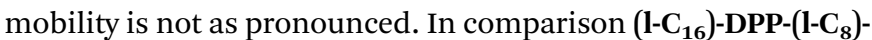

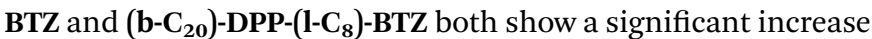
in saturated hole and electron mobility when annealed at temperatures of up to $300{ }^{\circ} \mathrm{C}$ which could be caused by the need to eliminate specific trapping sites and/or to enhance thin-film ordering and therefore lower reorganization energy by annealing at high temperatures for these polymer synthesized by DARP.
As shown by GIWAXS measurement of films of (1-C $\left.\mathbf{C}_{\mathbf{1 8}}\right)$-DPP$\left(\mathbf{b C}_{\mathbf{1 7}}\right)$-BTZ at different annealing temperatures (see Fig. S3†) the

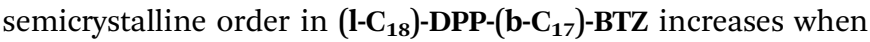
annealed at higher temperatures. However no related increase in hole mobilities can be observed suggesting that the film morphology at $110{ }^{\circ} \mathrm{C}$ annealing temperature is already ordered enough to enable the highest possible degree of charge-trans-

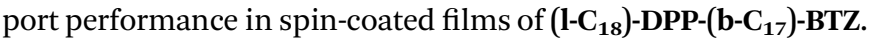

To gain a deeper insight into the charge transport of the studied polymers, temperature dependent measurements were performed on transistors annealed at temperatures ensuring optimal transistor performance (i.e. $300{ }^{\circ} \mathrm{C}$ for $\left(\mathbf{l}-\mathbf{C}_{\mathbf{1 6}}\right)$-DPP-(1-

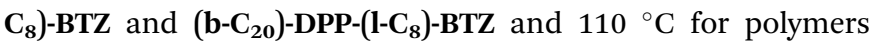

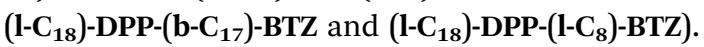

Fig. $\mathrm{S} 4 \uparrow$ shows the dependence of the extracted saturated

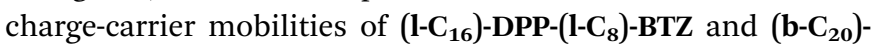
DPP-(1-C $\left.\mathbf{C}_{\mathbf{8}}\right)$-BTZ as a function of measurement temperature. The carrier mobilities show a simple Arrhenius-like, temperature activated behavior as demonstrated by the linear dependence of mobility on a $1 / \mathrm{T}$ scale. Activation energies for holes and electrons of $135 \mathrm{meV}$ and $141 \mathrm{meV}$ for $\left(\mathbf{1 - C _ { 1 6 }}\right)-\mathbf{D P P}-\left(\mathbf{l}-\mathbf{C}_{\mathbf{8}}\right)-\mathbf{B T Z}$ and

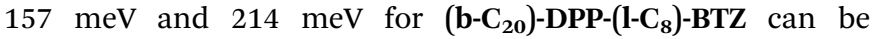
extracted. The decreased activation energy for charge-carrier

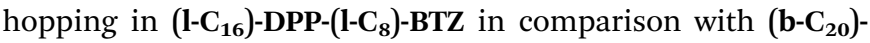
DPP-(1-C $\left.\mathbf{C}_{\mathbf{8}}\right)$-BTZ is consistent with the increased charge-carrier mobilities. As expected from the increased mobility and the higher degree of structural order, the activation energy for charge-transport in $\left(\mathbf{1}-\mathbf{C}_{\mathbf{1 8}}\right)$-DPP-(1-C $\left.\mathbf{8}\right)$-BTZ with values of $100 \mathrm{meV}$ for holes and electrons is even smaller than for $\left(\mathbf{1}-\mathbf{C}_{\mathbf{1 6}}\right)$ DPP-(1-C 8 )-BTZ.

In contrast to $\left(\mathbf{1}-\mathbf{C}_{\mathbf{1 8}}\right)$-DPP-(1-C $\left.\mathbf{C}_{\mathbf{8}}\right)-\mathbf{B T Z}$, the low-temperature

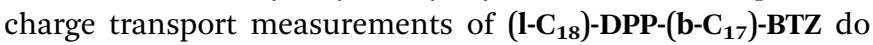
not show a simple Arrhenius behavior with just one slope. The Arrhenius plot of saturated hole-mobility over $1 / \mathrm{T}$ shown in Fig. 5 shows two clearly distinguishable regions with different slopes in the temperature regime ranging from 300 down to $120 \mathrm{~K}$ with a change of temperature behavior at around $250 \mathrm{~K}$ for $\left(\mathbf{l}-\mathbf{C}_{\mathbf{1 8}}\right)$-DPP-(b-C $\left.\mathbf{C}_{\mathbf{1 7}}\right)$-BTZ. In the high temperature region above $250 \mathrm{~K}$ a high activation energy of $180 \mathrm{meV}$ is extracted, while in the low temperature region below $250 \mathrm{~K}$ a much lower activation energy value of around $65 \mathrm{meV}$ is observed suggesting different charge-transport mechanisms in the two regimes. The charge-transport activation energy value of $180 \mathrm{meV}$ extracted from the high temperature regime is unusually high for a high mobility polymer, as other high mobility polymers like PBTTT, DPP-BT or PSeDPP-BT usually show much lower activation energies in the range of $50-90 \mathrm{meV} .^{\mathbf{4 , 3 4 , 3 5}}$

A similar temperature dependent transport behavior with two distinct activation energy regimes was already proposed by Street et al. in 2005 for semicrystalline films of PQT-12. ${ }^{36}$ In this publication Street et al. explained the two different activation energy regions by the activation of charge transport through the disordered grain boundary regions at higher temperatures leading to an increase in activation energy. Recently Noriega et al. extended this model to explain the exceptionally high charge-carrier mobility values of poorly ordered polymers like DPP-BT by intrachain transport along long polymer tie-chains 


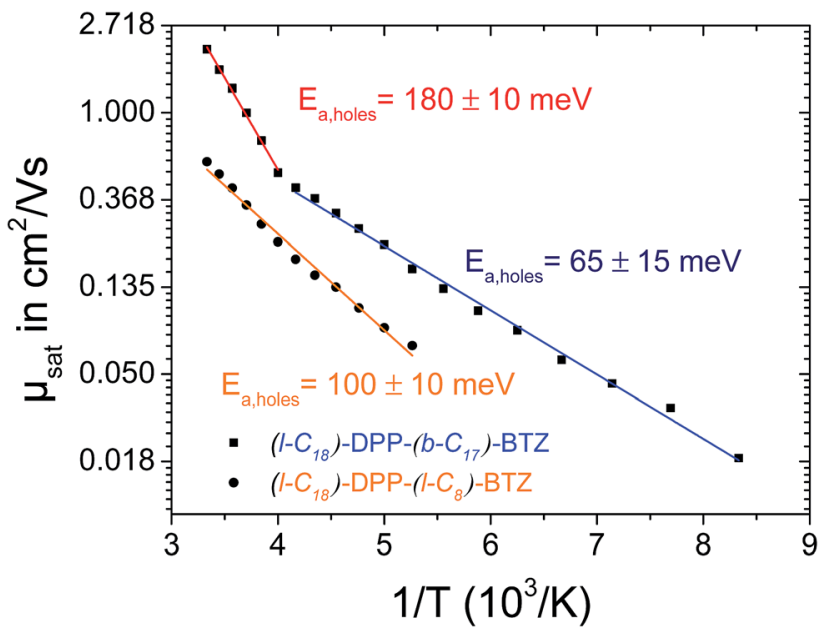

Fig. 5 Arrhenius plot of temperature dependent $p$-type field effect mobility of $\left(\mathrm{l}-\mathrm{C}_{18}\right)$-DPP-(b-C $\left.\mathrm{C}_{17}\right)-\mathrm{BTZ}$ (squares) and (l- $\left.\mathrm{C}_{18}\right)$-DPP- $\left(\mathrm{l}-\mathrm{C}_{8}\right)$ BTZ (circles). The blue, the red and the orange curves show linear fits for the activation energy extraction in the high and low temperature region (above and below $250 \mathrm{~K}$ ) for $\left(\mathrm{l}-\mathrm{C}_{18}\right)$-DPP- $\left(\mathrm{b}-\mathrm{C}_{17}\right)$-BTZ (red/ blue) and across the whole temperature range for $\left(\mathrm{l}-\mathrm{C}_{18}\right)$-DPP- $\left(\mathrm{l}-\mathrm{C}_{8}\right)-$ BTZ (orange). All measurements were taken in forward and reverse direction to.

bridging the disordered regions between small semicrystalline domains..$^{35} \mathrm{~A}$ comparable model based on percolation transport model can be used to explain the observed temperature

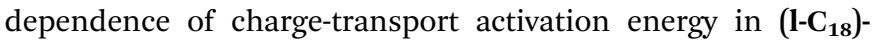
DPP-(b-C 17 )-BTZ.

We propose that charge-transport in (1-C $\left.\mathbf{C}_{\mathbf{1 8}}\right)$-DPP- $\left(\mathbf{b}-\mathbf{C}_{\mathbf{1 7}}\right)-\mathbf{B T Z}$ at temperatures below $250 \mathrm{~K}$ is dominated by a few percolation paths leading through more ordered regions of the film and mainly containing low-activation energy hops. At temperatures above $250 \mathrm{~K}$ the higher thermal energy enables additional transport paths to occur through somewhat more disordered regions of the polymer thin film increasing the measurable activation energy to values close to activation energies usually observed in purely amorphous polymers like regioregularamorphous-P3HT. ${ }^{37}$ Interestingly the same behaviour is not

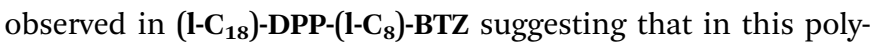
mer the difference in energy between the few percolation paths that allow charge transport at low temperature and the transport paths through more disordered regions is too large for the latter to become thermally accessible near room temperature.

\section{Conclusions}

We prepared four new low band-gap DPP-based co-polymers with BTZ to allow for additional solubilizing side-chains on the BTZ co-monomer. We used two different synthetic routes, direct arylation and Suzuki-polycondensation, and found from a comparison of polymers with only linear side chains made by the two routes that direct arylation produces a higher density of chain defects which manifest themselves as low band gap trap sites in UVVIS spectroscopy and lead to a lower electrical performance. We showed that the introduction of a linear side- chain on the DPP-unit leads to an increase in thin-film order and charge-carrier mobility provided that the BTZ co-monomer is substituted with a sufficiently solubilizing branched side

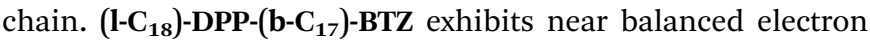
and hole mobilities; the average hole mobility of $2.4 \mathrm{~cm}^{2} \mathrm{~V}^{-1} \mathrm{~s}^{-1}$ is exceptionally high, in fact it is one of the highest, robustly extracted values reported for a DPP copolymer in a top-gate configuration to date. Our results demonstrate clearly that linear side chain substitution of the DPP unit is an attractive route for improving the charge transport properties of DPP copolymers. It requires the use of co-monomers however, that allow attachment of sufficiently long or branched side chains to retain sufficient solubility for solution processing. The experimental study presented here demonstrates very clearly that for a given conjugated polymer backbone judicious selection of the side chain substitution is crucial to ensure optimum device performance.

One of the challenges from a practical design point of view is that it is difficult to predict from mere reasoning or simple molecular modelling how a particular side chain substitution is likely to influence the polymer microstructure in the solid state. A microscopic understanding of the sensitive dependence of microstructure on side chain substitution generally requires sophisticated molecular dynamics modelling that goes beyond the scope of the present work. ${ }^{10,38}$ However, as such detailed calculations will be performed in the future on more high mobility polymers, such as the ones reported here, general molecular design guidelines for side chain substitution are likely to emerge. Our work suggests that copolymers with linear side chains on the DPP unit and branched side chains on the co-monomer may be an attractive design motif for high mobility DPP copolymers.

\section{Acknowledgements}

The authors gratefully acknowledge funding from the Engineering and Physical Sciences Research Council (EPSRC) through a program grant (EP/G060738/1). J.K.L. and S.H.J. thank the National Research Foundation of Korea (Grant No. 2011-0012274) funded by the Korean government (MEST). C.R.M. acknowledges support from the Australian Research Council (FT100100275). This research was undertaken in part on the SAXS/WAXS beamline at the Australian Synchrotron, Victoria, Australia. C.R.M. thanks Dr Nigel Kirby of the Australian Synchrotron for technical assistance and Dr Eliot Gann for assistance with data analysis.

\section{Notes and references}

1 (a) W. Hu, Y.-T. Tao and H. Sirringhaus, Phys. Chem. Chem. Phys., 2012, 14, 14097; (b) M. Jung, J. Kim, J. Noh, N. Lim, C. Lim, G. Lee, J. Kim, H. Kang, K. Jung, A. D. Leonard, J. M. Tour and G. Cho, IEEE Trans. Electron Devices, 2010, 57, 571; (c) H. N. Tsao, D. M. Cho, I. Park, M. R. Hansen, A. Mavrinskiy, D. Y. Yoon, R. Graf, W. Pisula, H. W. Spiess and K. Müllen, J. Am. Chem. Soc., 2011, 133, 2605. 
2 H. Chen, Y. Guo, G. Yu, Y. Zhao, J. Zhang, D. Gao, H. Liu and Y. Liu, Adv. Mater., 2012, 24, 4618.

3 (a) K.-J. Baeg, D. Khim, D.-Y. Kim, S.-W. Jung, J. B. Koo, I.-K. You, H. Yan, A. Facchetti and Y.-Y. Noh, J. Polym. Sci., Part B: Polym. Phys., 2011, 49, 62; (b) H. Yan, Y. Zheng, R. Blache, C. Newman, S. Lu, J. Woerle and A. Facchetti, Adv. Mater., 2008, 20, 3393; (c) B. Yoo, B. A. Jones, D. Basu, D. Fine, T. Jung, S. Mohapatra, A. Facchetti, K. Dimmler, M. R. Wasielewski, T. J. Marks and A. Dodabalapur, Adv. Mater., 2007, 19, 4028.

4 A. J. Kronemeijer, E. Gili, M. Shahid, J. Rivnay, A. Salleo, M. Heeney and H. Sirringhaus, Adv. Mater., 2012, 24, 1558.

5 (a) J. Lee, A.-R. Han, J. Kim, Y. Kim, J. H. Oh and C. Yang, J. Am. Chem. Soc., 2012, 134, 20713; (b) T. Lei, J.-H. Dou, Z.-J. Ma, C.-H. Yao, C.-J. Liu, J.-Y. Wang and J. Pei, J. Am. Chem. Soc., 2012, 134, 20025; (c) A. J. Kronemeijer, E. Gili, M. Shahid, J. Rivnay, A. Salleo, M. Heeney, H. Sirringhaus, Z. Bao and I. McCulloch, Proc. SPIE, vol. 8478, Organic Field-Effect Transistors XI, 847809.

6 Z. Chen, M. J. Lee, R. Shahid Ashraf, Y. Gu, S. Albert-Seifried, M. Meedom Nielsen, B. Schroeder, T. D. Anthopoulos, M. Heeney, I. McCulloch and H. Sirringhaus, Adv. Mater., 2012, 24, 647.

7 T.-J. Ha, P. Sonar, S. P. Singh and A. Dodabalapur, IEEE Trans. Electron Devices, 2012, 59, 1494.

8 (a) P. Sonar, S. P. Singh, Y. Li, M. S. Soh and A. Dodabalapur, Adv. Mater., 2010, 22, 5409; (b) J. Li, Y. Zhao, H. S. Tan, Y. Guo, C.-a. Di, G. Yu, Y. Liu, M. Lin, S. H. Lim, Y. Zhou, H. Su and B. S. Ong, Sci. Rep., 2012, 2, 754; (c) B. Sun, W. Hong, Z. Yan, H. Aziz and Y. Li, Adv. Mater., 2014, 26, 2636-2642, 2613; (d) I. Kang, H.-J. Yun, D. S. Chung, S.-K. Kwon and Y.-H. Kim, J. Am. Chem. Soc., 2013, 135, 14896.

9 C. B. Nielsen, M. Turbiez and I. McCulloch, Adv. Mater., 2013, 25, 1859.

10 Y. Olivier, D. Niedzialek, V. Lemaur, W. Pisula, K. Müllen, U. Koldemir, J. R. Reynolds, R. Lazzaroni, J. Cornil and D. Beljonne, Adv. Mater., 2014, 26, 2119.

11 (a) J. Mei and Z. Bao, Chem. Mater., 2014, 26, 604; (b) T. Lei, J.-Y. Wang and J. Pei, Chem. Mater., 2014, 26, 594; (c) T. Lei, J.-H. Dou and J. Pei, Adv. Mater., 2012, 24, 6457; (d) P. Sonar, T. Ru Bao Foong and A. Dodabalapur, Phys. Chem. Chem. Phys., 2014, 16, 4275.

12 (a) I. Osaka, R. Zhang, J. Liu, D.-M. Smilgies, T. Kowalewski and R. D. McCullough, Chem. Mater., 2010, 22, 4191; (b) X. Guo, F. S. Kim, S. A. Jenekhe and M. D. Watson, J. Am. Chem. Soc., 2009, 131, 7206.

13 X. Zhang, L. J. Richter, D. M. DeLongchamp, R. J. Kline, M. R. Hammond, I. McCulloch, M. Heeney, R. S. Ashraf, J. N. Smith, T. D. Anthopoulos, B. Schroeder, Y. H. Geerts, D. A. Fischer and M. F. Toney, J. Am. Chem. Soc., 2011, 133, 15073.

14 (a) J. Y. Back, H. Yu, I. Song, I. Kang, H. Ahn, T. J. Shin, S.-K. Kwon, J. H. Oh and Y.-H. Kim, Chem. Mater., 2015, 27, 1732; (b) C. Kanimozhi, N. Yaacobi-Gross, E. K. Burnett, A. L. Briseno, T. D. Anthopoulos, U. Salzner and S. Patil, Phys. Chem. Chem. Phys., 2014, 16, 17253.
15 T. L. Nelson, T. M. Young, J. Liu, S. P. Mishra, J. A. Belot, C. L. Balliet, A. E. Javier, T. Kowalewski and R. D. McCullough, Adv. Mater., 2010, 22, 4617.

16 J.-K. Lee, M. C. Gwinner, R. Berger, C. Newby, R. Zentel, R. H. Friend, H. Sirringhaus and C. K. Ober, J. Am. Chem. Soc., 2011, 133, 9949.

17 B. Fu, J. Baltazar, A. R. Sankar, P.-H. Chu, S. Zhang, D. M. Collard and E. Reichmanis, Adv. Funct. Mater., 2014, 24, 3734 .

18 B. Peng, A. Najari, B. Liu, P. Berrouard, D. Gendron, Y. He, K. Zhou, Y. Zou and M. Leclerc, Macromol. Chem. Phys., 2010, 211, 2026.

19 N. M. Kirby, S. T. Mudie, A. M. Hawley, D. J. Cookson, H. D. T. Mertens, N. Cowieson and V. Samardzic-Boban, J. Appl. Crystallogr., 2013, 46, 1670.

20 D. Apitz, R. Bertram, N. Benter, W. Hieringer, J. Andreasen, M. Nielsen, P. Johansen and K. Buse, Phys. Rev. E: Stat., Nonlinear, Soft Matter Phys., 2005, 72, 036610.

21 D. W. Breiby, O. Bunk, J. W. Andreasen, H. T. Lemke and M. M. Nielsen, J. Appl. Crystallogr., 2008, 41, 262.

22 J. D. Yuen, J. Fan, J. Seifter, B. Lim, R. Hufschmid, A. J. Heeger and F. Wudl, J. Am. Chem. Soc., 2011, 133, 20799. 23 (a) M. Karakus, D. H. Apaydın, D. E. Yıldız, L. Toppare and A. Cirpan, Polymer, 2012, 53, 1198; (b) L. Zhang, C. He, J. Chen, P. Yuan, L. Huang, C. Zhang, W. Cai, Z. Liu and Y. Cao, Macromolecules, 2010, 43, 9771; (c) X. Liu, P. Cai, Z. Chen, L. Zhang, X. Zhang, J. Sun, H. Wang, J. Chen, J. Peng, H. Chen and Y. Cao, Polymer, 2014, 55, 1707; (d) B. Liu, Y. Zou, S. Ye, Y. He and K. Zhou, RSC Adv., 2011, 1, 424; (e) S. Yum, T. K. An, X. Wang, W. Lee, M. A. Uddin, Y. J. Kim, T. L. Nguyen, S. Xu, S. Hwang, C. E. Park and H. Y. Woo, Chem. Mater., 2014, 26, 2147.

24 J.-K. Lee, H. H. Fong, A. A. Zakhidov, G. E. McCluskey, P. G. Taylor, M. Santiago-Berrios, H. D. Abruña, A. B. Holmes, G. G. Malliaras and C. K. Ober, Macromolecules, 2010, 43, 1195.

25 (a) J.-R. Pouliot, L. G. Mercier, S. Caron and M. Leclerc, Macromol. Chem. Phys., 2013, 214, 453; (b) Q. Guo, J. Dong, D. Wan, D. Wu and J. You, Macromol. Rapid Commun., 2013, 34, 522.

26 (a) L. G. Mercier and M. Leclerc, Acc. Chem. Res., 2013, 46, 1597; (b) K. Okamoto, J. Zhang, J. B. Housekeeper, S. R. Marder and C. K. Luscombe, Macromolecules, 2013, 46, 8059; (c) S. Kowalski, S. Allard, K. Zilberberg, T. Riedl and U. Scherf, Prog. Polym. Sci., 2013, 38, 1805; (d) A. E. Rudenko and B. C. Thompson, J. Polym. Sci., Part A: Polym. Chem., 2015, 53, 135; (e) Y. Fujinami, J. Kuwabara, W. Lu, H. Hayashi and T. Kanbara, ACS Macro Lett., 2012, 1, 67.

27 F. Lombeck, H. Komber, S. I. Gorelsky and M. Sommer, ACS Macro Lett., 2014, 3, 819.

28 (a) W. Lu, J. Kuwabara, T. Iijima, H. Higashimura, H. Hayashi and T. Kanbara, Macromolecules, 2012, 45, 4128; (b) A. Luzio, D. Fazzi, F. Nübling, R. Matsidik, A. Straub, H. Komber, E. Giussani, S. E. Watkins, M. Barbatti, W. Thiel, E. Gann, L. Thomsen, C. R. McNeill, M. Caironi and M. Sommer, Chem. Mater., 2014, 26, 6233; 
(c) S. Kowalski, S. Allard and U. Scherf, ACS Macro Lett., 2012, 1, 465.

29 K. H. Hendriks, W. Li, G. H. L. Heintges, G. W. P. van Pruissen, M. M. Wienk and R. A. J. Janssen, J. Am. Chem. Soc., 2014, 136, 11128.

30 A. B. Tamayo, M. Tantiwiwat, B. Walker and T.-Q. Nguyen, J. Phys. Chem. C, 2008, 112, 15543.

31 M. Caironi, M. Bird, D. Fazzi, Z. Chen, R. Di Pietro, C. Newman, A. Facchetti and H. Sirringhaus, Adv. Funct. Mater., 2011, 21, 3371.

32 (a) H. Bronstein, Z. Chen, R. S. Ashraf, W. Zhang, J. Du, J. R. Durrant, P. S. Tuladhar, K. Song, S. E. Watkins, Y. Geerts, M. M. Wienk, R. A. J. Janssen, T. Anthopoulos, H. Sirringhaus, M. Heeney and I. McCulloch, J. Am. Chem. Soc., 2011, 133, 3272; (b) R. S. Ashraf, Z. Chen, D. S. Leem, H. Bronstein, W. Zhang, B. Schroeder, Y. Geerts, J. Smith, S. Watkins, T. D. Anthopoulos, H. Sirringhaus, J. C. de Mello, M. Heeney and I. McCulloch, Chem. Mater., 2011, 23, 768 .
33 (a) Y. Li, S. P. Singh and P. Sonar, Adv. Mater., 2010, 22, 4862; (b) H.-W. Lin, W.-Y. Lee and W.-C. Chen, J. Mater. Chem., 2012, 22, 2120.

34 C. Wang, L. H. Jimison, L. Goris, I. McCulloch, M. Heeney, A. Ziegler and A. Salleo, Adv. Mater., 2010, 22, 697.

35 R. Noriega, J. Rivnay, K. Vandewal, F. P. V. Koch, N. Stingelin, P. Smith, M. F. Toney and A. Salleo, Nat. Mater., 2013, 12, 1038.

36 R. Street, J. Northrup and A. Salleo, Phys. Rev. B: Condens. Matter Mater. Phys., 2005, 71, 165202.

37 S. Ukai, H. Ito, K. Marumoto and S.-i. Kuroda, J. Phys. Soc. Jpn., 2005, 74, 3314.

38 D. Venkateshvaran, M. Nikolka, A. Sadhanala, V. Lemaur, M. Zelazny, M. Kepa, M. Hurhangee, A. J. Kronemeijer, V. Pecunia, I. Nasrallah, I. Romanov, K. Broch, I. McCulloch, D. Emin, Y. Olivier, J. Cornil, D. Beljonne and H. Sirringhaus, Nature, 2014, 515, 384. 